\title{
Research on the Influence of In-work Embedding on Employees' Constructive Deviant Behavior
}

\author{
Jiajun $\mathrm{Gu}^{1}$, Bang Cheng ${ }^{1, *}$ \\ ${ }^{1}$ School of Business Administration, Zhejiang Gongshang University, Hangzhou, Zhejiang310016, China
}

\begin{abstract}
This paper takes the identity theory as the theoretical background, adopts the empirical research method, takes the embedded work of employees as the antecedent, studies the constructive deviant behaviour of employees, and explores the intermediary mechanism of the identity perception of insiders from the perspectives of role identity. This research use SPSS25.0 version and Mplus 7.0 to analyse the date, some conclusions are as follows: 1) Employees' embeddedness in work has a significant positive effect on constructive deviant behavior.2) In-work embedding improves the role identity of employees, and has a significant positive impact on employees' perception of insider identity.3) Insider identity perception play a intermediary role between employees' embeddedness in work (in-work link, in-work match, in-work sacrifice) and constructive deviant behaviour. Starting from the perspective of double identity, this study has enriched the research on constructive deviant behaviour and provided beneficial reference for organizations to give play to the constructive power of employees in practice.
\end{abstract}

\section{Introduction}

The way of enterprise management is the way of talents. Under the current organization and management, it is the problem that enterprises are facing to give better play to the constructive power of talents. Enterprises need employees who can not only comply with norms to complete organizational tasks in conventional situations, but also have innovative vision to leap out of organizational norms and actively seek better solutions. The constructive behaviour of employees in an organization can help find problems in the development of the organization, but the constructive behaviour of employees may also involve violation of the existing norms of the organization ${ }^{[1]}$.

This reality makes scholars pay attention to this kind of extra-role behavior which is beneficial to the development of organizations but may violate the norms of organizations. The state embedded in the work makes employees more willing to stay in the current organization and creates a dependency on the organization. Under the severe external environment of current employment situation, it is of certain practical significance to discuss the role of in-work embedding for employees to play a constructive role.

Identity theorists put forward the study of interaction relationship between people and society can better explain the individual social behavior, to influence society by influencing the individual identity, individual behavior identity between social structures and individual behavior plays a conduction effect, and people to define themselves by identity, from the identity theory found on the concept of role identification, character identification is achieved by identity standards define the process of self.

Therefore, based on the identity theory, this paper integrates role and personal identity into the study of behavior, and derives a conceptual model for the study of employee constructive deviant behavior.

\section{Research design}

\subsection{Research hypothesis}

\subsubsection{Research hypothesis of work embeddedness on constructive deviant behavior}

Constructive deviant behavior is a kind of special role behavior, its behavior with the two-sided of for and against organizations, employee behavior purpose is to promote the development of the organization, but its behavior also standardize the devastating to the organization, from the Angle of employees, this behavior has certain risks, need big driving force to propel the individual to produce this behavior.

Previous studies mainly studied constructive deviant behaviours through organizational or leadership factors, such as high-quality leader member exchange relationship, inclusive leadership, high commitment work system, etc. This paper directly studied the impact of employees' embedding degree in work on constructive deviant behaviours from the perspective of employee status. Links to a high degree of employee in the organization should be given more emotional support and human resources, it provides a safe environment for our employees awareness,

\footnotetext{
*Corresponding author: chengbang198@163.com
} 
on the one hand, can to some extent employee deviant behavior generated by psychological stress, on the other hand would encourage employees to make constructive behavior in order to obtain more resources and interests.

The match between individuals and organizations enables employees to integrate into the organizational environment more quickly, and they believe that individuals can make a difference in the organization, and they are more willing to engage in behaviours outside their roles. For example, employees will put forward innovative ideas to improve the situation of the organization. Sacrifice increased awareness can make the employees have a higher dependence to organization, employees are more willing to attached to the organization, and the organization has a higher sense of belonging to employees will be willing to engage in constructive deviant behavior to promote the development of the organization, from the perspective of the employee organization development will inevitably increase the interests of individuals, employees will choose to bear the pressure to seek interests.

Based on the above analysis, this study puts forward the following hypotheses:

H1: In-work embeddedness has a positive influence on constructive deviant behavior.

\subsubsection{Research hypothesis of job embedding on the perception of insider identity}

Based on the theory of identity, this paper proposes that role identity is a process in which individuals define themselves according to a certain identity standard, and individuals define themselves by demarcating and naming social categories. In addition, identity will make individuals distinguish relevant roles and provide a sense of meaning for individuals.

In-work embeddedness is the product of a good interactive relationship between employees and organizations. Hom et al. (2009) found in their research that the social exchange relationship between employees and organizations can strengthen the embeddedness of employees' work, which is the product of a good relationship ${ }^{[2]}$. In addition, high job embeddedness can improve employees' emotional commitment, increase their identification with the organization, and reduce turnover intention. Insider identity perception measure whether the employees consider themselves is organization insider, under the background of Chinese culture, the others will pass the individuals with factors such as resources and trust as its identity, the distinction between inside and outside to individuals, resources and trust given by individuals to perceive themselves as a group of insiders, organization of employees to assume the role of trust resources way passed to the staff, employees perceive our insider status is of self in the expression of the identity of the roles in your organization, also gives new meaning to self-role.

Therefore, insider identity perception can be used as an important variable to measure the role identity of employees.
Embedded staff will make the staff and the organization produces network link, this intimate contact of the psychological make employees perceive the importance of the role that the individual in the organization, promotion role identity. Job matching increases the role identification of employees and makes them have a high emotional attachment and belonging to the organization and classifies themselves as an insider of the organization. The resources provided by the organization meet the needs of employees for resources, and effectively play a motivational factor. The incentive effect of resources will make employees satisfied with their role in the organization and have a high sense of attachment, so it will improve the insider perception of employees. In-work embedding influences employees' internal identity perception through resource state.

Based on the above analysis, the following hypotheses are proposed:

$\mathrm{H} 2$ : In-work embedding has a positive impact on insider identity perception.

\subsubsection{The mediator role of insider identity perception}

Based on the theory of identity, identity is an important factor to predict employee behavior. Stryker (1980) proposed that the role generated in every kind of society has the participation of self-component, that is, the generation of role identity ${ }^{[3]}$. In the interaction between people and society, different roles will come into being. The identification of individuals to roles can help individuals realize self-reference and self-definition and complete the process of self-cognition.

Employees embedded in the organizational network will increase the link between employees and the organization, the perceived job matching and the increase of turnover sacrifice cost, which will make employees more willing to stay in the current organization, and generate identification and emotional commitment to the organization. This kind of identification will enable employees to derive an insider's identity perception, and this psychological perception will make employees feel more emotional attachment in the organization, and make employees willing to do the behavior conducive to the organization.

Based on the above analysis, the following hypotheses are proposed in this study:

H3: Insiders' identity perception plays a mediating role between work embeddedness and constructive transgression.

The theoretical model of this paper is shown in Figure 1.

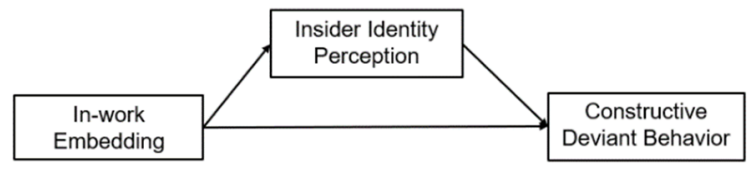

Figure 1 Research model 


\subsection{Methods}

\subsubsection{Samples}

Based on the theory of identity, combined with the embedded within the work for constructive deviant behavior research background, the existing literature to find a mature scale to measure the relevant variables, before the formal questionnaire measurement, first on a small scale questionnaire investigation, and to examine reliability and validity of scale and to correct the problem - item, ultimately determines the formal issuing questionnaires, this study questionnaire must working fixed number of year of the personnel of different industries.

In this survey, males accounted for $41.7 \%$ and females accounted for $58.3 \%$, the ratio of males was lower than that of females. Secondly, in terms of the age distribution of this survey, respondents aged 25-30 account for the largest proportion of $47.6 \%$, followed by respondents aged below 25 accounting for $22.5 \%$, respondents aged $30-35$ accounting for $18.5 \%$, and people aged over 40 accounting for $4.8 \%$.In real life, the current labour market is mainly dominated by young people, and the sample data certainly shows the trend of younger labour market in real life. In terms of educational background, bachelor's degree accounts for the largest proportion, accounting for $57.6 \%$, and more than half of the samples have bachelor's degree, followed by master's degree, accounting for $19.2 \%$. Junior college degree and doctor's degree account for less, accounting for 3.3\% and $2.6 \%$ respectively. In terms of the nature of the company, private enterprises have the largest number of employees, accounting for $55 \%$, followed by state-owned enterprises, accounting for $17.3 \%$. The distribution of foreign enterprises and government agencies is less, and the distribution of research is wider, which is also more in line with the distribution of actual company types.

In terms of working years, the number of samples is evenly distributed in four stages: less than 3 years, 3-5 years, 5-10 years, and more than 10 years, but $70.8 \%$ of them have worked in the current company for less than 3 years.

Through the above descriptive statistical analysis, the sample of this survey has good representativeness and meets the requirements of the study. In this chapter, statistical software SPSS24.0 and MPLUS 7.0 will be used to analyse the large sample data.

\subsubsection{Measures}

The design of the in-work embeddedness scale was based on the questionnaire of Mitchell and Lee (2001) ${ }^{[4]}, 14$ items were determined to measure the three dimensions of in-work linkage, in-work matching and in-work sacrifice. By measuring in-work link with 3 items, in-work matching with 5 items and in-work sacrifice with 6 items, reliability analysis shows that the Cronbach's value of the scale is 0.865 , AVE is 0.50 and CR is 0.93 .

In the research on internal identity perception, the 6item one-dimensional measurement scale of Stamper and
Masterson (2002) ${ }^{[5]}$ was used, among which 3 items were positive measurement and the other 3 items were negative measurement. Reliability analysis showed that Cronbach's value of the scale was 0.918, AVE was 0.51, and CR was 0.86 .

Constructive deviant behavior has three dimensions: innovation, challenge and interpersonal. This article USES the Galperin (2002) ${ }^{[6]}$ the use of three dimension scale, a total of 16 item five questions for measuring innovative constructive deviant behavior, 6 item type measurement challenges constructive deviant behavior, 5 item type measuring interpersonal constructive deviant behavior, reliability analysis show that the scale of the Cronbach 's value of 0.881 , AVE 0.53 , CR is 0.94 .

All the scales are measured by Likert's 5-point scoring method, where 1 means very different and 5 means very agree.

\section{Result}

\subsection{Correlation analysis}

The correlation analysis showed that embedding in work is positively correlated with insider identity perception $(\mathrm{r}=0.482, \mathrm{p}<0.01)$ and constructive deviant behavior $(\mathrm{r}=0.616, \mathrm{p}<0.01)$. In addition, there is a significant positive correlation between insider identity perception and constructive deviant behavior $(\mathrm{r}=0.413, \mathrm{p}<0.01)$.

The results of correlation analysis among variables show that the correlation between job embeddedness and insider identity perception and constructive deviant behavior has reached a significant level, which lays a foundation for testing the regression relationship in the next step.

\subsection{Regression analysis}

In this paper, SPSS24.0 was used to test the relevant hypotheses, and the test method in this paper was hierarchical regression.

According to Model 2, in-work embedding has a significant positive effect on insider identity perception $(\beta$ $=0.582>0, \mathrm{P}<0.01)$, that is, $\mathrm{H} 2$ is supported. According to Model 3, in-work embeddedness has a significant positive effect on constructive deviant behavior $(\beta=0.599>$ $0, \mathrm{P}<0.01$ ), that is, hypothesis $\mathrm{H} 1$ is true. By model 6 , after joining intermediary variable insider identity perception, perception and constructive insider identity of deviant behavior standardized regression coefficient is $0.022 \mathrm{~b}(\mathrm{p}>0.01)$, embedded within work of constructive aberration coefficient $\mathrm{c}$ was $0.599(\mathrm{p}<0.01)$, shows that insider identity perception of intermediary effect, $\mathrm{H} 3$ was supported. 


\section{Conclusion}

\subsection{Main research conclusions}

Based on the theory of identity, this paper uses the empirical research method to study the constructive deviant behavior of employees by taking the work embeddedness as the antecedent and explores the mediating mechanism of insider identity perception from the perspective of role identity. This paper draws the following research conclusions:

(1) In-work embedding can positively influence employees' constructive deviant behaviours. Embedded deep in this study found that job resources and the relationship of the employees have eased the pressure generated by employee behavior, first of all, the embedded deep contact with existing staff members degree is high, this kind of network connection make employees have more resources and emotional support, such as good relations between employees and other members of the organization, employees directly against other people's thinking and behavior, reduced the interpersonal conflict, tissue compatibility makes employees more likely to engage in role higher external behavior, leaving perception of high cost, make employees are more willing to stay in the organization, In addition, employees will have a stronger perception of the community of interests between themselves and the organization, so as to promote employees to engage in pro-organization behavior.

In the current economic development situation, the organization is facing product demand and market innovation and technology update quickly iteration big challenge, so organizations need to employees can achieve greater innovation, so that the organization can get good position in the market, and embedded within the work can make produce self-interests and organization of the combination of perception, so that employees are more actively engaged in activities to achieve organizational interests, in this paper, we study expand the constructive deviant behavior of former research.

(2) In-work embedding will enable employees to have role identification and enhance the identity perception of insiders. Embedded in the work is from the economic and emotional comprehensive perception of individual situation measured variables, is to organize the product of a good relationship with members, embedded high work staff and other members of your organization has the close relation, the responsibilities of the expectations and the actual match, fit and organizational values, and once to leave the current position, there will be a big cost loss, under the combined action, employees will produce good feedback to the role of self, the role of employees will produce high identity, the role of identity, through insider staff itself is divided into groups.

(3) Constructive deviant behavior embedded in work without the role of insider's identity perception. The results showed that job embeddedness could not influence employees' constructive deviant behaviours through single role identification, and on the other hand, role identification had insufficient driving force on employees' behaviours. The author thinks that the results have some reasons. First, under embedded within the working staff with insider identity perception, employee identity role in the organization itself, but the employee pressure to make constructive deviant behavior is influenced by other factors, such as staff identity, staff awareness of the organization's deeper personal profit factors, such as the identity of the insiders have enough to affect employee breaches of organizational behavior, work within embedded in a constructive and other transmission mechanism between the deviant behavior. Secondly, the insider identity can make the employees have an emotional attachment to organization, the attachment may make employees to organization rules and relationships, such as employee in the face of some deviate from the conventional innovation behavior can take a more cautious attitude, so the identity of the insiders can't drive the staff to make constructive deviant behavior.

\subsection{Research enlightenment}

Empirical studies have found that the role of the embedded inside work cannot through the staff identity affect constructive deviant behavior, role identity through personal identity to affect positive deviant behavior, when organizations face change and can improve the staff's personal identity rather than the role identification, cultivate positive innovation talents within the organization contribute to the development of organizational change.

On the one hand, enterprises should encourage employees to develop high connections and promote their sense of self-identity. The enterprise should establish a variety of formal and informal communication channels for employees, strengthen the communication within the department and between departments, to ensure the healthy development of employee relations. In terms of environment, enterprises can establish an open space environment to reduce internal barriers and increase opportunities for communication between employees. Especially for new employees, they should arrange them in a more open place. In terms of atmosphere, the system and corporate culture should be adopted to create a harmonious working atmosphere. The internal competition among employees should not be overemphasized, and the working concept of cooperation and win-win should be emphasized.

By means of job rotation, transfer and training, the matching degree of people and posts and values can be improved to promote employees to have a high selfidentity in the organizational atmosphere. At the recruitment and training stage, specific measures should be taken to increase the matching degree of employees to the organization through recruitment and screening. In the process of selecting and organizing employees, the human resources department should not only pay attention to the suitability of posts, but also pay attention to the matching degree of values between employees and the enterprise, and take the matching degree of values as a condition to be included in the process of personnel screening and assessment. For fresh employees, to take the way of post 
rotation to determine the most suitable position and achieve the match of people and posts. For employees recruited by the society, their performance in the position they applied for should be evaluated. If there are positions that do not match with them, similar positions can be replaced.

We should actively authorize, make good use of incentives, set up challenging work contents, and improve employees' work initiative and sense of efficacy. Employees will have more initiative in their work, which will promote their enthusiasm, and they will be better involved in their work. The organization should actively empower employees so that they can make full use of their knowledge and skills to complete the work. With the more input of employees, the work will bring deeper meaning to employees, and employees will be more willing to treat the work as their own career to complete it. Reasonable setting of work content should be carried out instead of immutable work content. Work content should be more challenging. Only when faced with challenges can individuals give full play to their potential and gain a sense of efficacy from constant challenges.

When employees complete their tasks perfectly, they should make good use of incentives to reward them and encourage them to continue to innovate in their work.

On the other hand, employees should be encouraged to break the hierarchical barriers, support their truthseeking attitude at work, and establish a fair and trusting working environment.

Challenging constructive deviant behavior refers to the behavior of violating organizational norms and boss's instructions to complete tasks in an organization. Interpersonal construction deviant behavior refers to the behavior of violating boss's instructions and pointing out others' mistakes to improve working procedures and promote the development of members.

Employees in order to solve the problem caused by violation of boss instruction, proposed colleagues mistake is worth advocating, as bridge water company founder ray Dali in author describes principles, support staff have open mind and honest attitude, employee to improve the company decision-making to other colleagues and even lead to opposition, this kind of "deviant behavior" can improve the company's decision, have a positive impact on organization.

Providing a fair and trusting work environment allows employees to be willing to break these norms and ask questions that will advance everyone.

Leaders should have equal dialogue and communication with employees, deal with the challenging and deviant behaviours of employees, reflect on the problems from themselves, and adopt a fair and just attitude to solve the problems, and break the restrictions and norms caused by hierarchy. Pay attention to employees' innovative deviant behaviours, and support and help reasonable innovation demands. Enterprises should pay attention to employees' innovative deviances. If a complete ban is adopted, employees' enthusiasm and sense of identity will be severely hit, and employees may have lazy behaviours.

Although the author carries out the research in this paper with a rigorous attitude, there are still many shortcomings. This section will discuss the shortcomings of this paper to improve and perfect it in the future research. First, this paper USES the data is the cross section data, this paper proves that the embedded within work through the role of the intermediary insider identity perception affect innovative deviant behavior, but ignore the work embedded within the psychology and behavior, which makes staff time factor, the longitudinal time data, employees can track discusses embedded within, insider identity perception, organizational psychological ownership and the relationship between the innovative deviant behavior, make the model more complete. Second, this article does not affect work embedded within the boundary of the employee deviant behavior constructive situation carries on the discussion, future research can add a moderator variable in the model, a clear organization of the work for embedded within situational factors affecting behavior, for example, the organization factors such as atmosphere, Chinese collectivism culture, makes the model more rich.

\section{References}

1. Spreitzer G M, Sonenshein S. Toward the Construct Definition of Positive Deviance[J]. American Behavioural Scientist, 2004, 47(6):828-847.

2. Hom P W, Tsui A S, Wu J B, et al. Explaining Employment Relationships With Social Exchange and Job Embeddedness[J]. Journal of Applied Psychology, 2009, 94(2):277-297.

3. Stryker, Symbolic Interactionism, A Social Structural Version[M]. Palo Alto: Benjamin Cummings, 1980, p385.

4. Mitchell, T. R., and Lee, T. W. The unfolding model of voluntary turnover and job embeddedness: foundations for a comprehensive theory of attachment[J]. Research in Organizational Behavior. 2001, (23) :189-246.

5. Stamper, C.L., Masterson, S-S-. Insider or Outsider? How Employee Perceptions of Insider Status Affect Their Work Behavior[J]. Journal of Organizational Behavior, 2002, 23(8):875-894.

6. Galperin B L. Determinants of deviance in the workplace: an empirical examination in Canada and Mexico[J]. John Molson School of Business, 2002. 